\title{
Chemical protective clothing; a study into the ability of staff to perform lifesaving procedures
}

\author{
Mark J Coates, Ayman S Jundi, Mark R James
}

\begin{abstract}
Objective-To investigate the ability of medical and nursing staff to perform certain tasks while wearing a chemical protection suit with a respirator. Tasks chosen were those that would be required before decontamination.

Methods-Ten experienced accident and emergency doctors (middle grade and consultants) and 10 nurses were asked to perform certain tasks that were judged to be life saving, relevant to triage, or necessary to confirm death, on an advanced life support manikin, while wearing a TSTSweden chemical protection suit. The operators were objectively assessed by one of the authors for achieving each task, then asked to make a subjective assessment of the difficulty experienced.
\end{abstract}

Results-Medical staff were asked to ventilate the manikin using a bag-valvemask, intubate within 30 seconds, apply monitor electrodes and cables and check cardiac rhythm, apply gel pads and defibrillate safely, and finally, fold the cruciform triage card to show "RED", and attach it to the manikin. All the doctors completed these tasks, except for one, who could only intubate the manikin after several attempts. Nursing staff were asked to open and apply an oxygen mask, adjust oxygen flow, size and insert an oropharyngeal airway, ventilate the manikin using a bag-valve-mask, apply a pressure bandage to a limb, and fold the cruciform triage card to show "YELLOw", and attach it to the manikin. All the nurses completed these tasks. Operators reported varying degrees of difficulty, the most difficult tasks were those requiring fine movements or delicate control. Generally, operators found the butyl rubber gloves cumbersome. Communication difficulties were frequently reported. Although only intubation was formally timed, tasks were perceived to take longer. Some operators found the suits too warm and uncomfortable.

Conclusion-Should the need arise, the TST-Sweden chemical protection suits would enable experienced doctors and nurses to perform lifesaving measures effectively, without significant impairment to their skills. Tasks would be easier to accomplish with better fitting gloves. (f Accid Emerg Med 2000;17:115-118)

Keywords: chemical incidents; protection suits; contaminated casualties
In 1997, a total of 931 "chemical incidents" were reported from hospitals, public health organisations, or other medical professional organisations, to the Chemical Incident Response Service (CIRS) ${ }^{1}$ at the Medical Toxicology Unit, Guy's and St Thomas' Hospital Trust. The majority of these figures (584) represents incidents identified by CIRS from hospital inquiries. A large number of these incidents affected multiple casualties, and some involved dealing with highly toxic chemicals.

The Ambulance Service Association has produced Interim Guidance on Chemical Incidents. ${ }^{23}$ Three triage categories were identified:

- P1: require resuscitation during, or before, decontamination.

- P2: treatment may be delayed until after decontamination on a stretcher.

- P3: may be decontaminated in ambulant facilities.

It is suggested that, for P1 category patients, only immediately lifesaving procedures should be performed before decontamination. ${ }^{4}$

The accident and emergency (A\&E) department at the Royal Preston Hospital is a designated receiving facility for casualties from a chemical incident. It has purchased a number of TST-Sweden chemical protection suits (Textil Skyddsteknik AB, Kinna, Sweden; distributed in the UK by Airshelta, Huddersfield; fig 1); each consists of a polyurethane coated polyamide overall (EN 465), and a butyl coated polyester hood (EN 146). Ventilation is provided by a battery operated blower unit and a filter that meets CEN class A2 (organic gases and vapours), B1 (inorganic gases and vapours), E2 (acidic gases and sulphur dioxide), K1 (ammonia and its organic derivatives), and P3 (toxic particles, bacteria, viruses). It comes with separate butyl rubber boots, and butyl rubber gloves. The suits are designed for dealing with contaminated casualties in a clean environment. They meet or exceed the recommendations of the Ambulance Service Association, ${ }^{23}$ and would provide protection against most agents encountered during decontamination of patients in a clean environment, including victims of nerve gas attack..$^{56}$ They are not, however, designed for use within the high risk contaminated exclusion zone, where only firefighters wearing chemical protection suits equipped with self contained breathing apparatus can operate.

In the course of staff training on chemical incident management in the $\mathrm{A} \& \mathrm{E}$ department at the Royal Preston Hospital, concern was expressed as to whether lifesaving procedures 


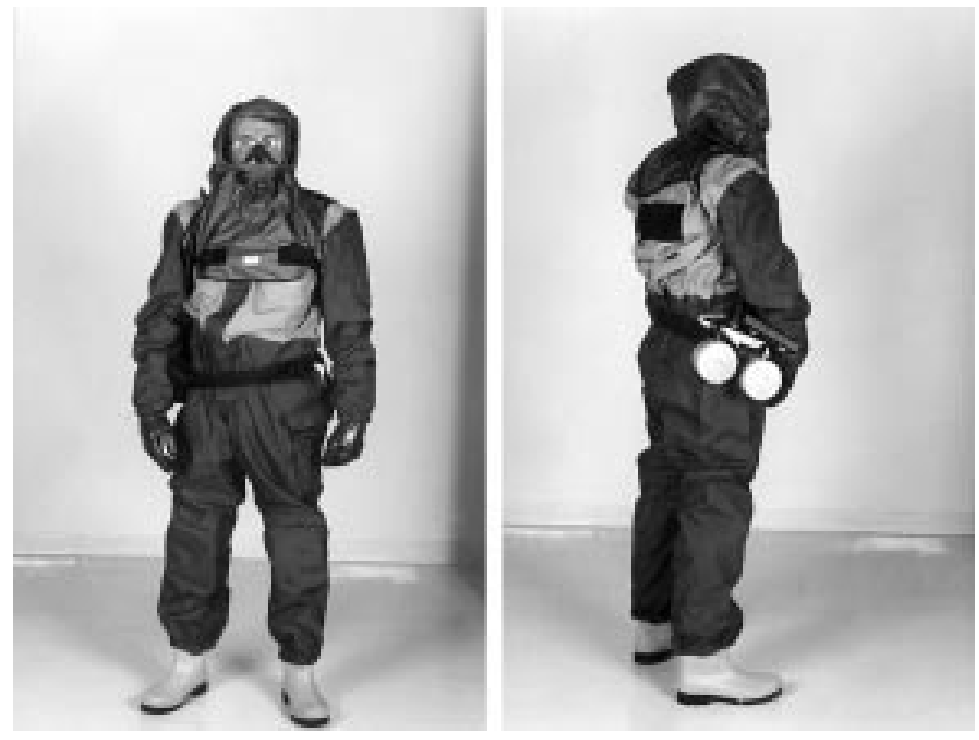

Figure 1 TST-Sweden chemical protection suit.

could be carried out safely and effectively while wearing the chemical protection suits that are available in the department.

The aim of the study was to establish whether medical and nursing staff would be capable of performing lifesaving procedures, or confirming death, while wearing the TSTSweden chemical protection suits. We also tried to convey staff perceptions of the difficulty of achieving these tasks while wearing the suits.

\section{Methods}

Twenty medical and nursing staff were invited to perform a number of tasks that were deemed immediately life saving, relevant to triage, or necessary to confirm death, while wearing the chemical protection suits. These tasks were selected to represent interventions that might be required before decontamination of P1 patients.

This was carried out as part of the A\&E department's ongoing training on chemical incident management.

Medical staff were asked to perform the following tasks:

- Ventilate the manikin using a bag-valvemask apparatus.

- Intubate the manikin within 30 seconds.

- Apply electrocardiographic monitor electrodes, connect the cables, and check cardiac rhythm.

- Fold the cruciform triage card to show "RED", starting from a standard preset position, reinsert it into its pouch, and attach it to the manikin.

- Open the gel pad packet, apply gel pads to the manikin, and defibrillate safely, using a Zoll PD 1400 defibrillator.

Nursing staff were asked to perform the following tasks:

- Remove a non-rebreathing oxygen mask from its packet, apply the mask to the manikin, connect the tubing to an oxygen source, and adjust the oxygen flow to $10 \mathrm{l} / \mathrm{min}$.

- Select, size, and insert an oropharyngeal airway.
- Ventilate the manikin using a bag-valvemask apparatus.

- Apply a pressure bandage to a forearm, using a 5 inch roll of wool, and a 5 inch roll of crepe bandage, then secure it using adhesive tape.

- Fold the cruciform triage card to show "YELLOw", starting from a standard preset position, reinsert it into its pouch, and attach it to the manikin.

All participants were also asked to feel for the carotid and radial pulses of the authors while wearing the butyl rubber gloves.

The operators were objectively assessed by one of the authors for achieving each task. Staff were then asked to make a subjective assessment of the difficulty experienced in performing these tasks, on a scale of 1 to 5, with 1 being as easy as performing the task without the suit, and 5 being so hard as to make the task unachievable. Staff were also given the opportunity to make general comments about the suits afterwards.

Staff were familiar with all the equipment used, with the exception of the chemical protection suit, and were not given the opportunity to practice any tasks in the suit.

\section{Results}

Ten doctors and 10 nurses participated in the study, each performing the required tasks in sequence, after putting on the chemical protection suit.

The first task given to the doctors was to ventilate an advanced life support (ALS) manikin using a bag-valve-mask apparatus. This was achieved by all 10 participants the mean difficulty rating being 1.8 (range $1-3$ ). The main difficulty was in sensing the effectiveness of the mask seal around the face of the manikin.

All but one middle grade doctor managed to intubate the manikin in the allocated time; a second middle grade doctor failed the first attempt, but managed the task on the second. This was scored as "achieved", as it would be considered a "pass" under ALS test conditions. The difficulty rating ranged from $2-5,5$ being given by the person who failed to achieve the task. The mean rating for this task was 2.8. Tying the endotracheal tube in place was reported as the most difficult part of this task.

Applying the electrodes to the chest, while achieved by all doctors, was found to be the most "fiddly" task, particularly peeling the back off the self adhesive electrodes. The mean difficulty rating was 3.7 (range 3-4). Folding the cruciform triage card and applying it to the manikin was successfully achieved by all participants, with a mean difficulty rating of 2.4 (range 2-3).

The final task, applying the gel pads and using the defibrillator safely, was also successfully achieved by all the doctors. It was, however, rated at a slightly higher rating, with a mean of 2.7 (range 2-4). Operators commented on the difficulty in opening the gel pad packets, the lack of tactile feel to determine paddle contact with the chest, and difficulty in communication to warn colleagues to stand 


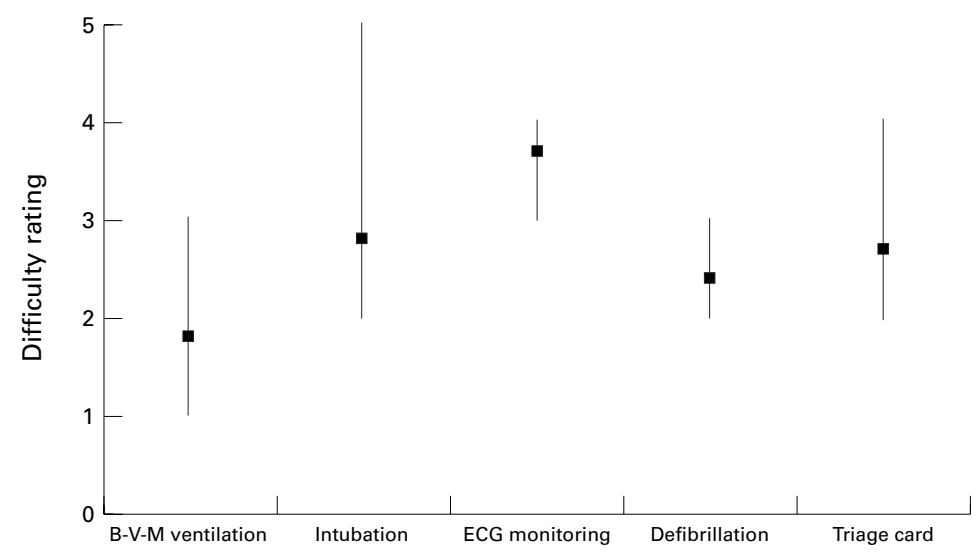

Figure 2 Means and ranges of difficulty ratings for tasks given to medical staff (B-V-M= bag-valve-mask; ECG = electrocardiography).

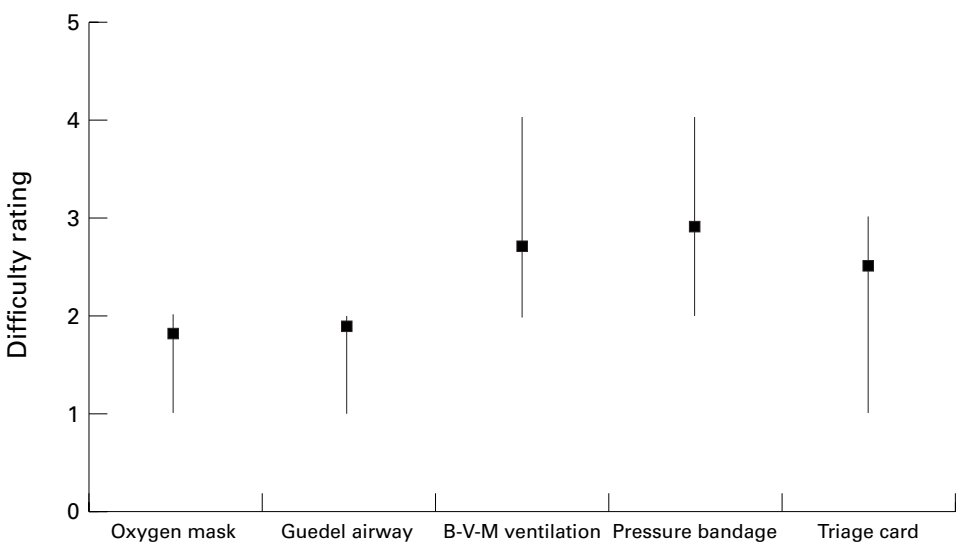

Figure 3 Means and ranges of difficulty ratings for tasks given to nursing staff (B-V-M= bag-valve-mask).

clear. Several participants experienced difficulty in hearing the change in the defibrillator's audible tone, indicating completion of charging. In general, the safety of nearby colleagues was an important concern for operators (fig 2).

The first task given to the nurses was to provide oxygen to the manikin at a flow rate of 10 $1 /$ min, through a non-rebreathing mask. All nurses could manage this task, with a mean difficulty rating of 1.8 (range 1-2). Sizing and inserting an oropharyngeal airway was, similarly, achieved by all; the mean difficulty rating being 1.9 (range 1-2).

All nurses managed to ventilate the manikin using a bag-valve-mask apparatus. The mean difficulty rating was 2.7 (range $2-4$ ). As was the case for the doctors, the main difficulty reported was the inability to sense the adequacy of the mask seal around the mouth of the manikin.

Applying a pressure bandage to the manikin's forearm was successfully carried out by all the participating nurses. The mean difficulty rating was 2.9 (range $2-4$ ). The main problem was pulling out the end of the adhesive tape used to secure the bandage. Folding and applying the cruciform triage card was performed successfully by all participants, with a mean difficulty rating of 2.5 (range $1-3$ ) (fig 3 ).

Participants were able to feel the carotid pulse of the authors, but two thirds of them found it impossible to feel the radial pulse.
Bearing in mind that the authors were, presumably, in a normal cardiovascular condition, it would be conceivable that detecting the pulse of a moribund, seriously injured casualty would be considerably more difficult.

All the tasks were essentially achievable. However, they varied in difficulty. This seemed to be related to the degree of fine movement and tactile feedback that were involved in the execution of the task. Most operators have expressed that they found the gloves to be too big and cumbersome, resulting in impairment of fine movement performance, and loss of tactile feedback. Communication difficulties were also frequently reported by participants, mostly due to background noise from the blower unit. Visual field restrictions also added to communication difficulties. Most participants felt that tasks took longer to achieve, and many felt that the suits became warm and uncomfortable, even in the relatively short time that they wore them.

\section{Discussion}

Chemical incidents pose a particular problem for the emergency services and health care workers. Casualties presenting for treatment might be a source of dangerous, or even fatal, contamination to staff. Casualties should always be decontaminated before treatment if their condition allows it, and they should be presented to the ambulance and hospital staff "as clean as possible". ${ }^{3}$ That, however, may not necessarily mean that they are totally safe to handle without protection. In addition, some casualties may be in urgent need of lifesaving treatment before they can be safely decontaminated. The adequate protection of staff, under these circumstances, must take the first priority, and the TST-Sweden suits can provide that protection, against most agents, in a non-contaminated atmosphere.

Several studies, mostly from the military medical literature, have looked at the physiological effects of wearing protective clothing for prolonged periods. ${ }^{7-9}$ Other studies have analysed exercise tolerance in soldiers wearing such clothing, ${ }^{10}{ }^{11}$ or the effects of such clothing on psychomotor and cognitive performance of troops. ${ }^{12}$ These studies have investigated such effects on army personnel trained to operate while wearing chemical protective suits almost as a matter of routine. One study looked at skill decay after prolonged wearing of protective clothing among army paramedics ${ }^{13}$ and suggested that while tasks took longer to achieve, the quality of the soldiers' performance did not suffer as a results of wearing the suits for eight hours. Again, this study looked at highly trained personnel used to working in chemical protection suits as part of their every day activities.

Our study looked at a small number of experienced medical and nursing staff, all from within our department. It showed that experienced medical and nursing personnel who may have to treat contaminated casualties while wearing chemical protection clothing can, to a large extent, perform most essential lifesaving procedures safely and effectively. It also 
showed that confirmation of death by assessing pulse is likely to be unreliable, and the use of a stethoscope is, clearly, impossible with the hood. On the other hand, it showed that operators can use a defibrillator/monitor to confirm the presence or absence of myocardial electrical activity when confirmation of death is required before decontamination of the casualty. However, the study highlights certain issues, such as the need for better fitting gloves. It also demonstrated that the alien environment of the suits imposes communication difficulties, mostly caused by the background noise from the blower unit and the auditory insulation caused by the hood. The hood also greatly restricted the visual field, making visual communication difficult as well. Staff need to be aware of these problems, and to take the necessary steps to minimise their effect. One interesting observation was that a small number of staff found the suits very uncomfortable, to the degree of experiencing claustrophobic feelings, and those staff members could not tolerate the suits for more than a few minutes. The study, and the ongoing training, helped identify those members of staff who found the suits intolerable, and allowed them to be assigned alternative tasks in the departmental chemical incident plan.

We did not make any attempts to test participants' ability to obtain venous access while wearing chemical protection suits. We felt that the cannulation manikins currently available are so unrealistic as to make any comparison with human subjects invalid. Plans are currently being considered for such studies on human volunteers, or stable patients requiring cannulation. Ethics committee approval and carefully laid out inclusion and exclusion criteria will be required for this.

No statistical analysis was carried out. We realise that in this study we have looked only at a small number of fairly experienced medical and nursing staff, and we appreciate that this may make it difficult to draw hard conclusions. However, we felt that chemical incidents of any significance would be managed primarily by senior staff, who are more likely to be able to manage critically ill patients in an unfamiliar environment. With that taken into consideration, we believe that the sample is representative and the findings valid in the setting described.

\section{Conclusion}

Should the need arise to treat contaminated casualties from a chemical incident, experienced medical and nursing staff wearing the
TST-Sweden chemical protection suits would be able to perform emergency lifesaving measures effectively, and without significant impairment to their skills. Further studies would be required to determine the feasibility of other essential procedures, such as obtaining venous access.

After communication of the above findings to TST-Sweden, they have informed us that the butyl rubber gloves will be available in a full range of glove sizes.

Contributors

Dr Mark Coates contributed to the conception of the original idea of the project and the selection of the tasks to be tested, helped design the data collection proforma, and participated in data collection. He also helped in revising the paper before submission for publication. Mr Ayman Jundi helped in the selection of the tasks to be tested, helped design the data collection proforma, carried out most of the data collection, carried out the analysis and interpretation of data, and the formulation of conclusions. He carried out the literature search and the writing of the paper. Mr Mark James contributed to the conception of the original idea of the project and the selection of the tasks to be tested. He helped in revising the paper before submission for publication.

Mr Mark James acts as a guarantor for the contents of the
publication. paper.

Conflict of interest: none.

Funding: none.

1 Medical Toxicology Unit, Guy's and St Thomas' Hospital Trust. Chemical incident report. No 7. London: Medical Toxicology Unit, Guy's and St Thomas' Hospital Trust, January 1998.

2 Chemical Incident Procedures Working Group. The Ambulance Service Association: interim guidance on chemical incidents. London: Ambulance Service Association, August 1996.

3 Walker RJ. The Ambulance Service Association: interim guidance on chemical incidents. Pre-Hospital Immediate Care 1997;1:137-43.

4 Advanced Life Support Group. Major incident medical management and support. A practical approach. London: BMJ Publishing Group, 1995.

5 Ministry of Defence. Medical manual of defence against chemical weapons. (JSP312.) 6th Ed. MoD D/Med $(F \& S)(2) / 10 / 1 / 1$. London: HMSO, 1987.

6 Volans AP. Sarin: guidelines on the management of victims of a nerve gas attack. F Accid Emerg Med 1996;13:202-6.

7 Muza SR, Banderet LE, Forte VA. Effects of chemical defence clothing and individual equipment on ventilatory function and subjective reactions. Aviat Space Environ Med 1996;67:1190-7.

8 McLellan TM, Frim J. Heat strain in the Canadian forces chemical defence clothing: problems and solutions. Can $\mathcal{F}$ Appl Physiol 1994;19:379-99.

9 McLellan TM, Jacobs I, Bain JB. Influence of temperature and metabolic rate on work performance with Canadian forces NBC clothing. Aviat Space Environ Med 1993;64: 587-94.

10 Aoyagi Y, McLellan TM, Shephard RJ. Effects of training and acclimation on heat tolerance in exercising men wearing protective clothing. Eur f Appl Physiol 1994;68:234-45.

11 White MK, Hodous TK. Reduced work tolerance associated with wearing protective clothing and respirators. $\mathrm{Am}$ Ind Hyg Assoc f 1987;48:304-10.

12 Fine BJ, Kobrick JL. Effect of heat and chemical protective clothing on cognitive performance. Aviat Space Environ Med 1987;58:149-54.

13 Arad M. Berkenstadt H. Zelingher J, et al. The effects of continuous operation in a chemical protective ensemble on the performance of medical tasks in trauma management. $\mathcal{F}$ Trauma 1993;35:800-4. 